\title{
REVIEW
}

\section{Myofascial Pain Syndrome: A Treatment Review}

\author{
Mehul J. Desai · Vikramjeet Saini $\cdot$ Shawnjeet Saini
}

To view enhanced content go to www.paintherapy-open.com

Received: September 27, 2012 / Published online: February 12, 2013

(c) The Author(s) 2013. This article is published with open access at Springerlink.com

\section{ABSTRACT}

Myofascial pain syndrome (MPS) is defined as pain that originates from myofascial trigger points in skeletal muscle. It is prevalent in regional musculoskeletal pain syndromes, either alone or in combination with other pain generators. The appropriate evaluation and management of myofascial pain is an important part of musculoskeletal rehabilitation, and regional axial and limb pain syndromes. This article reviews the current hypotheses regarding the treatment modalities for myofascial trigger points and muscle pain. Through a critical evidence-based review of the pharmacologic

M. J. Desai $(\bowtie) \cdot$ S. Saini

Pain Medicine and Non-Operative Spine Services, The George Washington University Medical Center, 2131K Street, NW Washington, DC 20037, USA e-mail: mdesai@mfa.gwu.edu

V. Saini

Medstar Georgetown University Hospital, NW Washington, DC 20007, USA

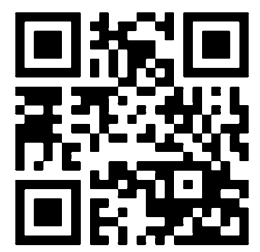

Enhanced content for this article is available on the journal web site: www.paintherapy-open.com and nonpharmacologic treatments, the authors aim to provide clinicians with a more comprehensive knowledge of the interventions for myofascial pain.

Keywords: Analgesics; Anticonvulsants;

Muscle relaxants; Myofascial pain;

Nonpharmacological treatment; Pain;

Treatment

\section{INTRODUCTION}

The conventional definition of myofascial pain syndrome (MPS) is characterized by regional pain originating from hyperirritable spots located within taut bands of skeletal muscle, known as myofascial trigger points (MTrPs) [1]. Common etiologies of myofascial pain and dysfunction may be from direct or indirect trauma, spine pathology, exposure to cumulative and repetitive strain, postural dysfunction, and physical deconditioning [2, 3]. Treating the underlying etiology is currently the most widely accepted strategy for MPS therapy. If the root cause is not properly treated, MTrPs may reactivate and MPS may persist [2]. 
Interestingly, there is a lack of specific diagnostic criteria for MPS. Electrodiagnostic and morphological findings have been identified; however, they cannot be practically applied in the clinical setting due to cost and time constraints. This adds to the difficulty of definitive treatment, particularly when considering elusive underlying pathology and persistent MTrPs.

The aim of this article is to examine the variety of treatments for MPS. Specifically, the evidence basis of pharmacological treatment and noninvasive therapy are reviewed. The purpose of this review is to provide the clinician with a comprehensive and up-to-date understanding of the current treatments for MPS.

\section{MATERIALS AND METHODS}

An extensive literature search was performed to create a comprehensive narrative in the treatments for myofascial pain. This was done by searching PubMed, Ovid, and Google Scholar for the key terms: <intervention of interest> and "myofascial pain" or "muscle pain." Controlled studies were given first priority, followed by observational studies. Systematic reviews and Cochrane reviews were included, and non-English sources were omitted. Given the considerable clinical overlap among musculoskeletal (MSK) disorders, e.g., fibromyalgia, regional soft-tissue pain, craniomandibular dysfunction, and tension headache, methods beneficial to an associated syndrome may prove useful in treating myofascial pain. In the absence of controlled data specifically examining drug efficacy in myofascial pain, the authors extrapolated from these associated disorders. Relevant studies with stronger levels of evidence were compiled and summarized for each method, and clinical recommendations were generated. It should be noted that the diagnostic criteria for MPS might vary between studies, so the conclusions drawn on the efficacy of various therapies must be taken with caution.

\section{PHARMACOLOGIC TREATMENT OF MYOFASCIAL PAIN}

\section{Analgesic Drugs}

Nonsteroidal anti-inflammatory drugs (NSAIDs) are the most commonly used drugs for MPS as they are readily available and have a relatively mild side-effect profile. Their use is appealing because of their analgesic and anti-inflammatory properties. Despite their widespread use, there are no randomized, controlled trials (RCTs) specifically evaluating oral NSAIDs in the treatment of MPS. Therefore, there is a lack of strong evidence for the role of an anti-inflammatory in MPS [4]. Multiple studies exist that demonstrate strong evidence in support of NSAIDs in treating acute MSK disorders, particularly low-back pain (LBP) [5-7].

Although the role of NSAIDs in treating MPS is unclear, there is clear evidence that the analgesic properties of NSAIDs relieve pain in acute MSK disorders [5-7]. With the considerable overlap between MPS and MSK pain, it would be reasonable to consider NSAIDs as an appropriate initial treatment in both disorders. However, long-term use should be considered with caution due to the gastrointestinal, renal, and antiplatelet side effects [6].

A diclofenac patch was evaluated in a RCT for myofascial pain of the trapezius muscle. There were statistically significant benefits with the diclofenac patch for pain $(P<0.01)$, cervical range of motion $(\mathrm{ROM})(P<0.01)$, and neck 
disability index $(P=0.03)$ by the end of treatment. The study demonstrated good patch tolerability and low overall skin irritation midway and at the end of the study [8].

Cyclooxygenase-2 (COX-2) inhibitors have an analogous effect to traditional NSAIDs with a relatively more-tolerable side-effect profile. Similar to NSAIDs, there are few supporting RCTs and evidence for their efficacy in MPS. Studies have shown that COX-2-selective inhibitors are an effective and well-tolerated agent for acute LBP $[9,10]$. Many studies have examined their effect on joint pain and chronic pain, which is outside the scope of this review. These agents should be considered in patients who are more prone to gastrointestinal side effects and do not have a history of thrombotic cardiovascular disease.

Tramadol is a centrally acting mu-receptor agonist, an inhibitor of dorsal horn presynaptic norepinephrine/serotonin reuptake, and increases central serotonin release. Studies have shown that tramadol is an effective and well-tolerated agent for use in LBP, some chronic pain syndromes, and osteoarthritis $[11,12]$. With regard to myofascial pain, there are no studies investigating the efficacy of this agent; however, it is used often for its multimodal analgesic effects and low abuse potential [11, 13-15].

Tropisetron is a $5-\mathrm{HT}_{3}$ receptor antagonist and alpha-7-nicotinic receptor agonist recently used as an analgesic for fibromyalgia and myofascial pain with a limited commercial availability. In a RCT, local injections of tropisetron at trigger points provided a statistically significant improvement in pain $(P=0.006)$ [16]. Its effects began rapidly and lasted longer than those of local anesthetics. Although this may appear a promising treatment for MPS, most of the available studies were written by the same group of authors, and thus, this requires further investigation.
Opioids are not normally indicated in the treatment for MPS. Although some limited studies demonstrate that weak opioids are moderately effective in the treatment of myofascial pain [17], most studies do not support the use of opioids in MPS. Furthermore, it is suggested that the use of opioids for the management of acute pain may actually be counterproductive to recovery [18].

The lidocaine patch is a transdermal local anesthetic preparation that alters the ability of nerves to conduct pain impulses [19]. A number of RCT studies, case reports, and observational studies examining the efficacy of lidocaine patches on MPS have been conducted. These studies were congruent in showing that lidocaine patches had a statistically significant increased pain thresholds $(P<0.001)$, and increase in general activity $(P<0.05)$ [20-22]. Topical lidocaine has shown promise as a therapy for MPS and is especially appealing as it is not an oral systemic drug.

\section{Muscle Relaxants}

Tizanidine is a centrally acting alpha-2-adrenergic agonist, which decreases muscle spasticity. An open-label, dose-titration study of tizanidine for MPS supported a significant decrease in pain intensity and disability from baseline $(P<0.01)$, and with improved sleep study periods [23]. Its efficacy in treating acute LBP has also been documented. Studies have suggested that tizanidine should be considered as a first-line agent for the treatment of MPS [23].

Benzodiazepines depress the presynaptic release of serotonin and excite gammaaminobutyric acid (GABA), which causes rapid inhibitory neurotransmission. In an open clinical trial, clonazepam was postulated to have an antinociceptive effect associated with 
MPS as it showed a statistically significant decrease in pain $(P<0.001)$ [24]. There are no RCTs evaluating the efficacy of clonazepam for MPS. Although there are limited data in the setting of MPS, a review of 30 RCTs was performed in 1997 to evaluate muscle relaxants on acute and chronic nonspecific LBP. The review showed that the use of muscle relaxant treatments is effective, but the associated adverse effects require that they be used with caution [6].

Cyclobenzaprine is another muscle relaxant that was recently studied in a Cochrane literature review. This review demonstrated a statistically significant decrease in mean pain intensity from baseline compared to clonazepam $(P=0.002) \quad[1]$. Another double-blind RCT compared cyclobenzaprine + ibuprofen versus placebo + ibuprofen in patients with acute myofascial pain [25]. There was no statistically significant difference in pain scores $(P=0.962)$ [25], but treated patients reported greater central nervous system side effects [25]. Cyclobenzaprine is efficacious as a muscle relaxant; however, there are no data to support treatment for MPS.

Thiocolchicoside (TCC) is a competitive $\mathrm{GABA}_{\mathrm{A}}$ antagonist and glycine agonist that also functions as an anti-inflammatory and analgesic [26], as well as a muscle relaxant [27]. A RCT in the setting of cervical myofascial pain demonstrated a statistically significant improvement in pain severity $(P<0.001)$ and ROM in those treated with topical TCC [28]. A double-blind RCT evaluating acute LBP associated with muscle spasm, supported the effectiveness of TCC and tizanidine over placebo in improving pain at rest, although tizanidine did cause somnolence [29]. There is a potential for TCC as a treatment in myofascial pain; however, there is limited evidence at this time.

\section{Anticonvulsants}

Gabapentin and pregabalin have analgesic, anxiolytic-like, and anticonvulsant activity, which reduces the release of several neurochemicals, including glutamate, noradrenaline, and substance P [30]. MPS may be mediated at the spinal level; therefore, anticonvulsants might be considered in its treatment [31]. To date, there are no RCTs of anticonvulsants in the treatment of MPS. A Cochrane literature review found that very few trials examined anticonvulsant effectiveness on acute pain; most examined their use in chronic pain [32]. A multicenter, double-blind RCT compared the effects of placebo with pregabalin on fibromyalgia. This demonstrated a significantly reduced average severity of pain in the pregabalin group and significantly more patients in this group had $>50 \%$ improvement in pain $(P=0.003)[30]$. Although anticonvulsants may have a therapeutic role in fibromyalgia, there is no evidence that they are effective for MPS and should be withheld until other interventions have been attempted [32].

\section{Antidepressants}

Tricyclic antidepressants (TCAs) are a class of medications that have been indicated for chronic pain, fibromyalgia, and neuropathic pain $[33,34]$. Their pain mitigating effects are not clear, but it is postulated that TCAs work on central serotonergic and noradrenergic signals, which affect central pain pathways [35]. Although their use is widespread, there are limited studies specifically for the treatment of MPS. A crossover study investigated the therapeutic effect of amitriptyline in treating chronic tension headaches and understanding its mechanism of action [36]. Compared to placebo, amitriptyline demonstrated a 
statistically significant reduction in tenderness $(P=0.01)$ and a highly significant reduction in headache intensity [36]. The study attributed this reduction to decreased neurotransmission of painful stimuli from the muscles rather than a general decrease in pain sensitivity [36]. Another study investigated amitriptyline use in the treatment of chronic temporomandibular disorder pain and showed a statistically significant reduction in all pain scores after 6 weeks of treatment (global treatment effectiveness, $P=0.007$ ) [37]. Currently, there is no indication for the use of these medications in the treatment of MPS; however, the growing body of evidence for their efficacy in chronic pain syndromes suggests an increased role in MPS when conventional treatments fail.

Duloxetine, a serotonin-norepinephrine reuptake inhibitor (SNRI), was recently found to be an evolving and possibly efficacious treatment for painful MSK conditions. As more research continues to be produced, this may prove to be especially useful in patients that also suffer from neuropathic pain or other mood disorders. A clinician must be vigilant of the numerous side effects of SNRIs (i.e., nausea, fatigue, diarrhea, hyperhidrosis, dizziness, constipation, and dry mouth) as well as its interaction with other medications [38].

Sumatriptan is a peripheral 5-HT receptor agonist commonly used for migraines. Data suggest that serotonin plays a role in the pathophysiology of myofascial pain [39]. A RCT examined its efficacy in reducing pain in patients with temporal muscle pain [40] and yielded a statistically significant reduction in pain intensity and increased pain relief $\left(P<1 \times 10^{-10}\right)[40]$. However, when compared to the placebo there was no significant difference $(P<0.57)$ [40]. This study demonstrated an effective use of sumatriptan; however, more studies are required before endorsing this is a mainstream treatment for MPS.

\section{Other Treatments}

Botulinum type A toxin (BoNT-A) is a potent neurotoxin that prevents muscle contraction. Its use in the treatment of muscle pain has been a topic of investigation recently and shows promise. It may have analgesic properties through decreased production of substance $\mathrm{P}$ and glutamate [41].

A recent, multicenter, prospective, randomized, double-blind study was conducted to evaluate the efficacy and tolerability of injections of BoNT-A at fixed locations in patients with moderate-to-severe myofascial pain of the upper back [42]. The study demonstrated a statistically significant difference in pain intensity, duration, and reduction of trigger points of the BoNT-A group $(P=0.001)$; however, these effects were seen after 4 weeks. An earlier study by the same group demonstrated similar results [43].

A Cochrane literature review that only included RCTs also evaluated BoNT-A in the setting of MPS. One of the four studies produced a statistically significant decrease in pain intensity scores and duration $(P<0.00001)$ [44]. Another literature review found one of five studies had a statistically significant benefit for treatment with BoNT-A $(P<0.01)$ [45]. Freund and Schwartz [46] found a statistically significant improvement in pain scores in patients treated with BoNT-A $(P<0.01)$. Lastly, when compared to saline injection, one study showed improvements in a visual analog scale; however, they were not statistically significant. Contrary to this evidence, two studies compared BoNT-A to placebo and showed no significant improvement in pain $[47,48]$. 
Finally, two studies compared BoNT-A to bupivacaine and BoNT-A to lidocaine trigger point injections, respectively $[49,50]$. Each treatment showed improvement but there was no significant difference between groups [49, 50].

The literature suggests that BoNT-A injection is a promising therapy to alleviate MPS, especially when it persists despite conservative treatment. Another appeal is that the side effects of muscle weakness and paralysis are transient, mostly local, and reversible [51].

Ketamine is a dissociative anesthetic, analgesic, and sedative that works as a noncompetitive $\mathrm{N}$-methyl-D-aspartic acid (NMDA) receptor antagonist. A RCT studied the use of intramuscular ketamine in the treatment of chronic myofascial pain in temporomandibular disorder. The study showed there was no statistically significant benefit to treatment with ketamine [52].

L-tryptophan and memantine are drugs that have been studied for the treatment of pain; however, these are older studies and there has been no recent research indicating their use in the treatment of myofascial pain [53].

A summary of pharmacologic treatments for myofascial pain can be found in Table $1[1,16$, $17,20-25,28,36,37,40,42-50,52,53]$.

\section{NONPHARMACOLOGIC TREATMENT OF MYOFASCIAL PAIN}

Injections into MTrPs are a common and effective treatment, presumably due to mechanical disruption by the needle and termination of the dysfunctional activity of involved motor endplates [39]. MTrP injections may employ dry needling, short- or long-acting anesthetics, or steroids.
Dry needling has been traditionally used as one of the fastest and most-effective ways to inactivate MTrPs and help alleviate the accompanied pain. The needle is placed into MTrPs using an in-and-out technique in multiple directions, in order to inactivate the MTrP. There are many studies investigating the effectiveness of dry needling; however, RCTs are difficult to design given the invasive nature of the procedure. In one study where patients underwent dry needling with and without lidocaine, it was concluded that both were effective in reducing MPS when eliciting local twitch responses during the procedure [54]. In another study with patients who were to undergo knee surgery, patients underwent dry needling or not while under general anesthesia and were evaluated postoperatively. This novel approach demonstrated significant a superiority of dry needling versus placebo in postsurgery analgesic demand $(P=0.02)$ and visual analog scores 1 month after surgery $(P<0.04)$ [55]. Several other studies by various authors have shown that dry needling is an effective treatment and equal in efficacy to trigger point injections, and should be used as the mainstay of acute treatment, despite complaints of postinjection soreness $[39,56]$.

Trigger point injections are similar to dry needling; however, they inject various solutions, typically a local anesthetic. Studies suggest similar efficacy to dry needling but with less discomfort [50]. A recent systematic review article on needling therapies for MTrPs found that, based on current medical evidence, the "nature of the injected substance makes no difference to the outcome and that wet needling is not therapeutically superior to dry needling" [57].

Steroid injections into MTrPs are controversial and without clear rationale 


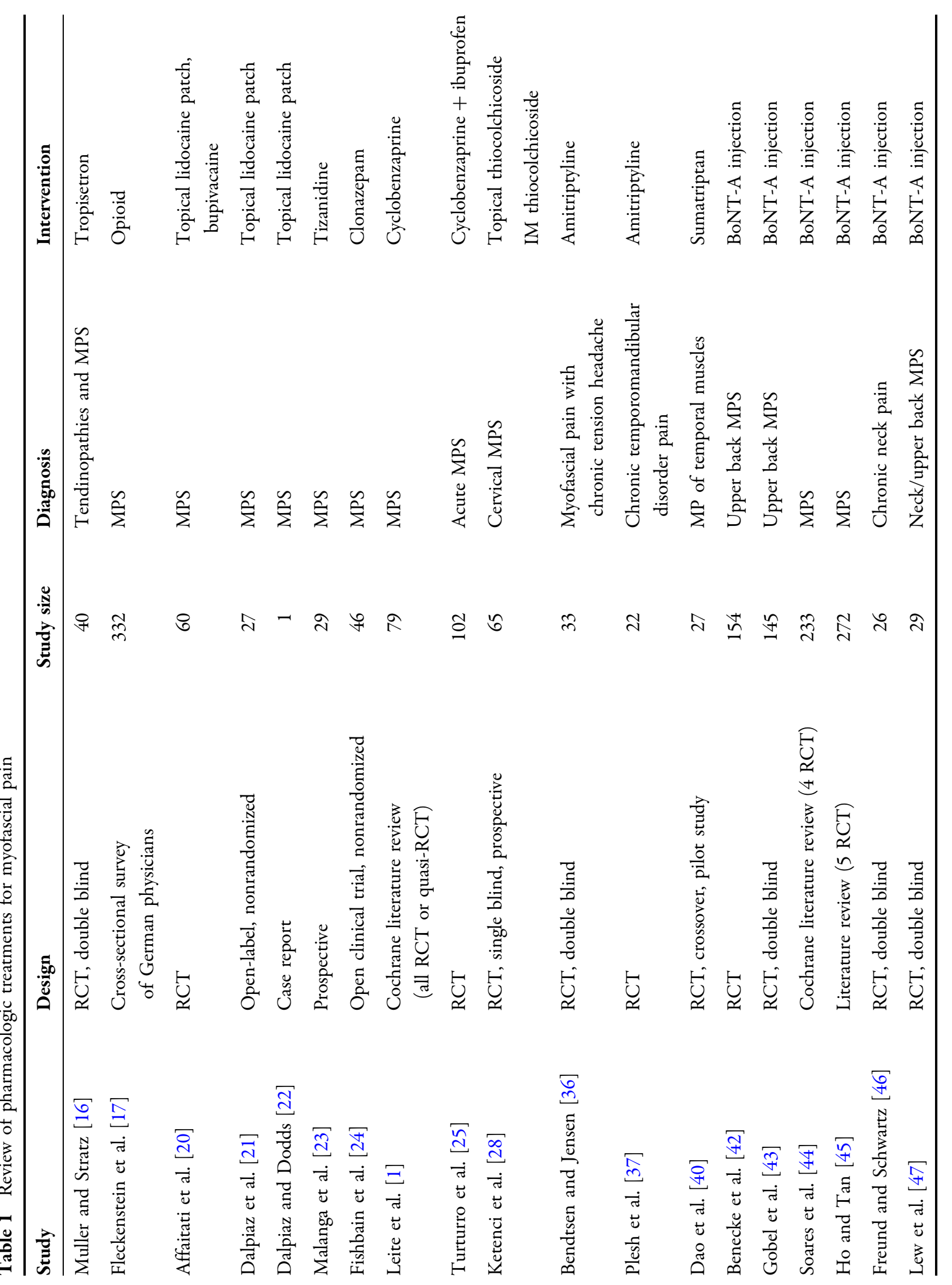






because little evidence exists to support an underlying inflammatory pathophysiology [39].

Manual therapy is a commonly used treatment for MPS as it has been considered as one of the most effective techniques for the inactivation of MTrPs $[2,58]$. There are various sources in the literature, which have specifically described effective modalities, including deep-pressure massage [59], stretch therapy with spray (where a taut band is stretched immediately after cold spray), superficial heat, and myofascial release [60]. Although there are many techniques that have been described in treating MPS with effective results, there have been no controlled studies proving significant long-term effectiveness.

Ultrasound is a technique that has been proposed to treat myofascial pain by converting electrical energy to sound waves in order to provide heat energy to muscles [61]. Multiple studies of ultrasound on MPS have been conducted; however, most demonstrate mixed results. A meta-analysis by Gam and Johannsen [62] reviewed 293 papers published since 1950 to assess the evidence for ultrasound in the treatment of MSK disorders. This found no documentation of effect; furthermore, the literature was generally of poor quality.

More recent RCTs have also been conducted. In one study on the treatment of latent MPS of the trapezius, ultrasound decreased the basal level of electrical activity and reduced the sensitivity of the trigger points [63]. Another RCT evaluating MTrP of the infraspinatus demonstrated a statistically significant increase in pain pressure threshold $(P<0.002)$ [64]. Another RCT compared ultrasound to diclofenac phonophoresis and placebo, which demonstrated a statically significant improvement in pain, ROM, number of trigger points, and neck pain disability index amongst the two former modalities $(P<0.05)$ [65]. There was no significant difference between the 
two treatment groups in those outcome measurements [65]. A similar study undertaken in 2012 examined the use of pressure release, hydrocortisone phonophoresis, ultrasound therapy, and placebo for the treatment of upper trapezius MTrPs. All three treatment groups demonstrated a statistically significant decrease in pain, and increase in pain threshold and ROM $(P<0.001)$ [66]. Pressure release and phonophoresis had superior therapeutic effects compared to ultrasound [66]. Likewise, another study concluded no statistically significant reduction in pain or analgesic usage between ultrasound + massage, sham ultrasound + massage, and exercise versus control [61].

The use of high-powered ultrasound has also been explored. A RCT study resulted in a statistically significant improvement in neck pain scores when compared to conventional ultrasound $(P<0.05)$ [67].

Transcutaneous electric nerve stimulation (TENS) is a treatment modality that utilizes an electrical current to stimulate nerve fibers in order to provide pain relief. TENS has been studied since 1970s and has recently been evaluated for the treatment of MPS [68]. One study in muscular face pain compared the use of TENS to biofeedback-enhanced relaxation and dental physiotherapy. It was shown that all three groups had equivalent results [68]. In a double-blind RCT, four modes of TENS were compared to controls and demonstrated that there was a significant reduction in pain with three of four modes: $100 \mathrm{~Hz}, 250 \mathrm{~ms} ; 100 \mathrm{~Hz}, 50 \mathrm{~ms}$; pain suppressor TENS $(P<0.001)$ [69]. Another RCT resulted in a statistically significant reduction in pain and increase in pain threshold versus control and electrical muscle stimulation $(P<0.01)$ [70]. TENS was further studied in another RCT that looked at TENS versus ultrasound versus TENS + ultrasound versus control. Ultrasound alone showed no significant benefit, whereas
TENS did show a reduction in pain immediately after therapy [71]. Another RCT looked at TENS versus frequency-modulated neural stimulation (FREMS) for the treatment of MPS [72]. It was shown that both TENS and FREMS had significant improvement in neck pain in the short term $(P<0.001)$ [72]. Finally, a 2009 RCT investigated TENS versus laser therapy versus lidocaine injection versus BoNT-A injection in patients with myofascial pain [73]. This study is one of the few to compare TENS to trigger point injections. It demonstrated no statistically significant benefit over injections [73]. Overall, TENS has been shown to have benefit among the noninvasive therapeutic modalities; however, there is currently no evidence that supports its use over trigger point injections or medication. At this point, TENS can be used as an adjuvant therapy to help alleviate MPS but should not be considered a monotherapy.

Electrical twitch obtaining intramuscular stimulation (ETOIMS) is another method of using an electrical current through a monopolar electromyography needle to engage deep motor endplates. Chu et al. [74] conducted separate studies on the use of ETOIMS specific to myofascial pain. A pilot study demonstrated a significant reduction in pain levels immediately after treatment in the ETOIMS group and these effects were sustained for 2 weeks posttreatment $(P<0.05)$ [74]. Another study in the setting of chronic refractory MPS resulted in significant improvement in immediate pain levels and ROM with no major side effects $(P<0.01)$ [75]. Furthermore, a significant negative correlation between number of treatments and pain level was noted $(P=0.00)$ [75]. ETOIMS has shown some potential as a treatment method; however, there is still a lack of RCTs; therefore, its use in MPS cannot be endorsed at this time.

Magnetic stimulation (MS) is a newer treatment that is being investigated for MSK 







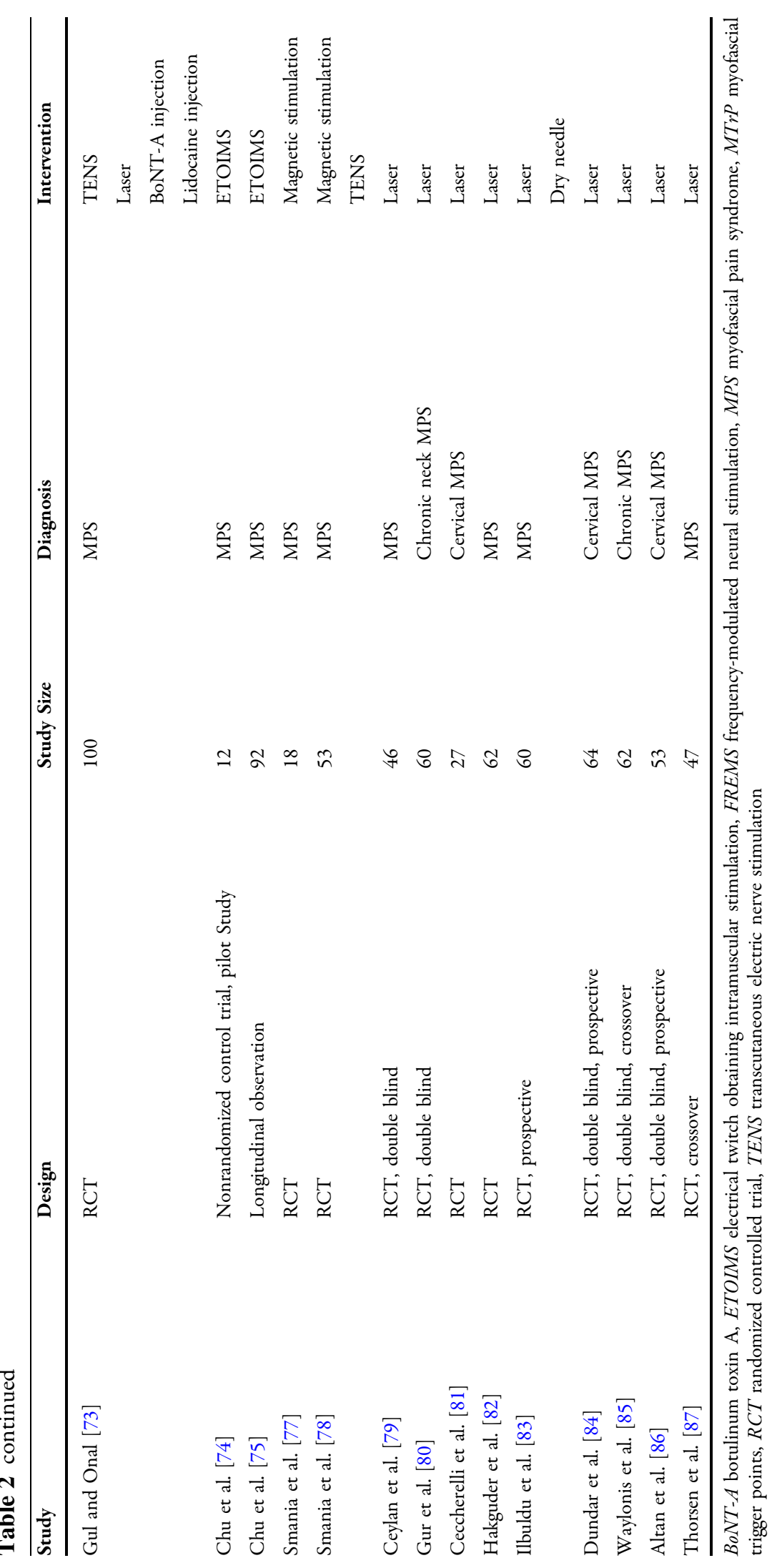


pain and MPS. Only a limited number of studies exist and the exact therapeutic mechanism of action remains uncertain [76]. In evaluating MS, one RCT concluded that it affords significant improvements in pain level $(P<0.01)$ and ROM $(P<0.035)$, and lasted for 1 month versus placebo [77]. Another RCT study compared MS to TENS and placebo. The results showed that MS had a statistically significant improvement in pain and ROM at 1 and 3 months after treatment, longer than the beneficial effects obtained after TENS $(P<0.05)$ [78]. MS has shown potential as a possible treatment method; however, more evidence is required before advising it as an efficacious treatment strategy.

Laser therapy has been used in the treatment of MSK pain including MPS; however, its exact mechanism of therapeutic action remains elusive. A 2004 RCT investigated laser versus placebo in the treatment of MPS and its effects on serotonin, a mediator of pain. Laser treatment demonstrated a statistically significant reduction in pain and increases in urinary excretion of serotonin degradation $(P<0.05)$ [79]. Multiple RCTs have determined a statistically significant improvement in pain with laser therapy $(P<0.01 ; P<0.002)[80,81]$. Yet another RCT study examined stretching with laser therapy and concluded that the laser treatment group had significantly decreased pain levels at the 3-week follow-up $(P<0.05)$ [82]. When comparing laser therapy with dry needling and placebo, a RCT showed that laser therapy significantly decreased overall pain and increased the pain threshold compared to the latter groups $(P<0.05)$ [83]. However, these benefits were no longer present at 6 months [83].

There is significant contradictory evidence for the benefit of laser therapy. A double-blind RCT investigated laser therapy versus placebo in the treatment of cervical myofascial pain. The authors concluded that there was no statistical difference between the two groups [84]. Another study analyzed laser therapy and found no significant benefit to its use [85]. Adding to this result, a 2005 study demonstrated that laser therapy did not have a significant benefit over placebo [86]. A 1992 study also concluded no beneficial effect for laser therapy in patients with myofascial pain [87]. Although laser therapy has shown some therapeutic promise, as a whole the body of evidence is mixed regarding the efficacy of this treatment strategy. At this point, the authors cannot endorse laser therapy as an appropriate treatment.

A summary of nonpharmacologic treatments for myofascial pain can be found in Table 2 [50, 54-57, 59-67, 69-75, 77-87].

\section{CONCLUSION}

This review of treatment in MPS finds that most interventions demonstrate a limited body of evidence for their use. This dearth of high-quality evidence is likely due to the heterogeneity of MPS combined with study design and methodologies. The treatment for MPS should focus primarily on identifying and correcting the underlying cause of the symptoms. The complex pathology of MPS with its underlying central and peripheral neural mechanisms may contribute to the difficulty in treating MPS, particularly in the chronic setting.

With regards to the pharmacologic treatments reviewed, tizanidine, benzodiazepines, and tropisetron appear to demonstrate some limited evidence for their use. Topical diclofenac and lidocaine patches may also have limited efficacy. TCC is a promising agent. There is evidence that NSAIDs and COX-2 inhibitors alleviate pain; however, more controlled trials are required to fully determine their role in MPS. 
Of the modalities reviewed, dry needling and trigger point injections are the mainstay of interventional treatment. Multiple studies support their use, but sustainability is likely based on using these therapies judiciously and in conjunction with manual therapies, such as myofascial release. Newer therapies, such as ultrasound and laser therapy, show promise.

Further research is needed to better establish algorithmic and evidence-based treatment of MPS.

\section{ACKNOWLEDGMENTS}

Dr. M. Desai is the guarantor for this article, and takes responsibility for the integrity of the work as a whole.

Conflict of interest. All authors declare no conflict of interest.

Open Access. This article is distributed under the terms of the Creative Commons Attribution Noncommercial License which permits any noncommercial use, distribution, and reproduction in any medium, provided the original author(s) and the source are credited.

\section{REFERENCES}

1. Leite F, Atallah A, El Dib R, et al. Cyclobenzaprine for the treatment of myofascial pain in adults. Cochrane Database Syst Rev. 2009;3:CD006830.

2. Simons D, Travell J, Simons L, editors. Travell and Simons' myofascial pain and dysfunction: the trigger point manual. 2nd ed. Baltimore: Williams \& Wilkins; 1999.

3. Wheeler A, Aaron G. Muscle pain due to injury. Curr Pain Headache Rep. 2001;5:441-6.

4. Fomby E, Mellion M. Identifying and treating myofascial pain syndrome. Phys Sport Med. 1997;25:67-75.
5. Lacey P, Dodd G, Shannon D. A double blind, placebo controlled study of piroxicam in the management of acute musculoskeletal disorders. Eur J Rheumatol Inflamm. 1984;7:95-104.

6. van Tulder M, Koes B, Bouter L. Conservative treatment of acute and chronic nonspecific low back pain: a systematic review of randomized controlled trials of the most common interventions. Spine (Phila Pa 1976). 1997;22:2128-56.

7. Amlie E, Weber H, Holme I. Treatment of acute low-back pain with piroxicam: results of a double-blind placebo-controlled trial. Spine (Phila Pa 1976). 1987;12:473-6.

8. Hsieh L, Hong C, Chern S. Efficacy and side effects of diclofenac patch in treatment of patients with myofascial pain syndrome of the upper trapezius. J Pain Symptom Manag. 2010;39:116-25.

9. Pohjolainen T, Jekunen A, Autio L. Treatment of acute low back pain with the COX-2-selective anti-inflammatory drug nimesulide: results of a randomized, double-blind comparative trial versus ibuprofen. Spine (Phila Pa 1976). 2000;25:1579-85.

10. Bosch H, Sigmund R, Hettich M. Efficacy and tolerability of intramuscular and oral meloxicam in patients with acute lumbago: a comparison with intramuscular and oral piroxicam. Curr Med Res Opin. 1997;14:29-38.

11. Borg-Stein J. Cervical myofascial pain and headache. Curr Pain Headache Rep. 2002;6:324-30.

12. Russell I, Kamin M, Bennett R. Efficacy of tramadol in treatment of pain in fibromyalgia. J Clin Rheumatol. 2000;6:250-7.

13. Borg-Stein J, Simons D. Focused review: Myofascial pain. Arch Phys Med Rehabil. 2002;83(Suppl 1):540-7.

14. Gerwin R. A review of myofascial pain and fibromyalgia-factors that promote their persistence. Acupunct Med. 2005;23:124-34.

15. Lewis K, Han N. Tramadol: a new centrally acting analgesic. Am J Health Syst Pharm. 1997;54:643-52.

16. Muller W, Stratz T. Local treatment of tendinopathies and myofascial pain syndromes with the $5-\mathrm{HT}_{3}$ receptor antagonist tropisetron. Scand J Rheumatol Suppl. 2004;119:44-8.

17. Fleckenstein J, Zaps D, Rüger L. Discrepancy between prevalence and perceived effectiveness of treatment methods in myofascial pain syndrome: results of a cross-sectional, nationwide survey. BMC Musculoskelet Disord. 2010;11:32. 
18. Webster B, Verma S, Gatchel R. Relationship between early opioid prescribing for acute occupational low back pain and disability duration, medical costs, subsequent surgery and late opioid use. Spine (Phila $\mathrm{Pa}$ 1976). 2007;32:2127-32.

19. Carterall W. Molecular mechanisms of gating and drug block of sodium channels. Sodium channels and neuronal hyperexcitability. Novartis Foundation Symposium 241; 2002.

20. Affaitati G, Fabrizio A, Savini A, et al. A randomized, controlled study comparing a lidocaine patch, a placebo patch, and anesthetic injection for treatment of trigger points in patients with myofascial pain syndrome: evaluation of pain and somatic pain thresholds. Clin Ther. 2009;31:705-20.

21. Dalpiaz A, Lordon S, Lipman A. Topical lidocaine patch therapy for myofascial pain. J Pain Palliat Care Pharmacother. 18:15-34.

22. Dalpiaz A, Dodds T. Myofascial pain response to topical lidocaine patch therapy: case report. J Pain Palliat Care Pharmacother. 2002;16:99-104.

23. Malanga G, Gwynn M, Smith R, Miller D. Tizanidine is effective in the treatment of myofascial pain syndrome. Pain Physician. 2002;5:422-32.

24. Fishbain D, Cutler R, Rosomoff H, Rosomoff R. Clonazepam open clinical treatment trial for myofascial syndrome associated chronic pain. Pain Med. 2000;1:332-9.

25. Turturro M, Frater C, D'Amico F. Cyclobenzaprine with ibuprofen versus ibuprofen alone in acute myofascial strain: a randomized, double-blind clinical trial. Ann Emerg Med. 2003;41:818-26.

26. Carta M, Murru L, Botta P, et al. The muscle relaxant thiocolchicoside is an antagonist of GABAa receptor function in the central nervous system. Neuropharmacology. 2006;51:805-15.

27. Biziere K, Huguet F, Narcisse G, Breteau M. Affinity of thiocolchicoside and thiocolchicoside analogues for the postsynaptic GABA receptor site. Eur J Pharmacol. 1981;75:167-8.

28. Ketenci A, Basat H, Esmaeilzadeh S. The efficacy of topical thiocolchicoside (Muscoril) in the treatment of acute cervical myofascial pain syndrome: a single-blind, randomized, prospective, phase IV clinical study. Agri. 2009;21:95-103.

29. Ketenci A, Ozcan E, Karamursel S. Assessment of efficacy and psychomotor performances of thiocolchicoside and tizanidine in patients with acute low back pain. Int $\mathrm{J}$ Clin Pract. 2005;59:764-70.

30. Crofford L, Rowbotham M, Mease $\mathrm{P}$, et al. Pregabalin for the treatment of fibromyalgia syndrome. Arthritis Rheum. 2005;52:1264-73.

31. Wheeler A. Myofascial pain disorders: theory to therapy. Drugs. 2004;64:45-62.

32. Wiffen P, Collins S, McQuay H, Carrol D, Jadad A, Moore A. Anticonvulsant drugs for acute and chronic pain. Cochrane Database Syst Rev. 2005;20(3):CD001133.

33. Micó J, Ardid D, Berrocoso E, Eschalier A. Antidepressants and pain. Trends Pharmacol Sci. 2006;27:348-54.

34. McQuay $H$, Tramèr $M$, Nye $B$, Carrol $D$, Wiffen $P$, Moore R. A systematic review of antidepressants in neuropathic pain. Pain. 1996;68:217-27.

35. Botney $M$, Fields $H$. Amitriptyline potentiates morphine analgesia by a direct action on the central nervous system. Ann Neurol. 1983;13:160-4.

36. Bendtsen L, Jensen R. Amitriptyline reduces myofascial tenderness in patients with chronic tension-type headache. Cephalalgia. 2001;20:603-10.

37. Plesh O, Curtis D, Levine J, McCall W Jr. Amitriptyline treatment of chronic pain in patients with temporomandibular disorders. J Oral Rehabil. 2000;10:834-41.

38. Smith H, Smith E, Smith B. Duloxetine in the management of chronic musculoskeletal pain. Ther Clin Risk Manag. 2012;8:267-77.

39. Borg-Stein J. Treatment of fibromyalgia, myofascial pain, and related disorders. Phys Med Rehabil Clin N Am. 2006;17:491-510.

40. Dao T, Lund P, Remillard G, Lavigne G. Is myofascial pain of the temporal muscles relieved by oral sumatriptan? A cross-over pilot study. Pain. 1995;62:241-4.

41. Fallah H, Currimbhoy S. Use of botulinum toxin A for treatment of myofascial pain and dysfunction. J Oral Maxillofac Surg. 2012;70:1243-5.

42. Benecke R, Heinze A, Reichel G, Hefter H, Gobel H, Dysport Myofascial Pain Study Group. Botulinum type A toxin complex for the relief of upper back myofascial pain syndrome: how do fixed-location injections compare with trigger point-focused injections? Pain Med. 2011;12:1607-14.

43. Göbel H, Heinze A, Reichel G, Hefter H, Gobel H, Dysport Myofascial Pain Study Group. Efficacy and 
safety of a single botulinum type A toxin complex treatment (Dysport) for the relief of upper back myofascial pain syndrome: results from a randomized double-blind placebo-controlled multicentre study. Pain. 2006;125:82-8.

44. Soares A, Andriolo R, Atallah A, da Silva E. Botulinum toxin for myofascial pain syndromes in adults. Cochrane Database Syst Rev. 2012;18:CD007533.

45. Ho K, Tan K. Botulinum toxin A for myofascial trigger point injection: a qualitative systematic review. Eur J Pain. 2007;11:519-27.

46. Freund B, Schwartz M. Treatment of whiplash associated neck pain [corrected] with botulinum toxin-A: a pilot study. J Rheumatol. 2000;27:481-4.

47. Lew H, Lee E, Castaneda A, Klima A, Date E. Therapeutic use of botulinum toxin type $A$ in treating neck and upper-back pain of myofascial origin: a pilot study. Arch Phys Med Rehabil. 2008;89:75-80.

48. Wheeler A, Goolkasian P, Gretz S. A randomized, double-blind, prospective pilot study of botulinum toxin injection for refractory, unilateral, cervicothoracic, paraspinal, myofascial pain syndrome. Spine (Phila Pa 1976). 1998;23:1662-6.

49. Graboski C, Gray D, Burnham R. Botulinum toxin A versus bupivacaine trigger point injections for the treatment of myofascial pain syndrome: a randomised double blind crossover study. Pain. 2005;118:170-5.

50. Kamanli A, Kaya A, Ardicoglu O, Ozgocmen S, Zengin F, Bayik Y. Comparison of lidocaine injection, botulinum toxin injection, and dry needling. Rheumatol Int. 2005;25:604-11.

51. Sławek J, Madaliński M, Maciag-Tymecka I, Duzynski W. Frequency of side effects after botulinum toxin A injections in neurology, rehabilitation and gastroenterology. Pol Merkur Lekarski. 2005;18:298-302.

52. Castrillon E, Cairns B, Ernberg M, et al. Effect of peripheral NMDA receptor blockade with ketamine on chronic myofascial pain in temporomandibular disorder patients: a randomized, double-blinded, placebo-controlled trial. J Orofac Pain. 2008;22:122-30.

53. Stockstill J, McCall W, Gross A, Piniewski B. The effect of L-tryptophan supplementation and dietary instruction on chronic myofascial pain. J Am Dent Assoc. 1989;118:457-60.

54. Hong C. Lidocaine injection versus dry needling to myofascial trigger point. The importance of the local twitch response. Am J Phys Med Rehabil. $1994 ; 73: 256-63$.

55. Mayoral del Moral O. Dry needling treatments for myofascial trigger points. J Musculoskelet Pain. 2010;18:411-6.

56. Edwards J, Knowles N. Superficial dry needling and active stretching in the treatment of myofascial pain: a randomised control trial. Acupunct Med. 2003;21:80-6.

57. Cummings T, White A. Needling therapies in the management of myofascial trigger point pain: a systemic review. Arch Phys Med Rehabil. 2001;82:986-92.

58. Kostopoulos D, Rizopoulos K. The manual of trigger point and myofascial therapy. New Jersey: SLACK Incorporated; 2001.

59. Hou C, Tsai L, Cheng K, Chung K, Hong C. Immediate effects of various physical therapeutic modalities on cervical myofascial pain and triggerpoint sensitivity. Arch Phys Med Rehabil. 2002;83:1406-14.

60. Hong C, Chen Y, Pon C. Immediate effects of various physical medicine modalities on pain threshold of an active myofascial trigger point. J Musculoskelet Pain. 1993;1:37-53.

61. Gam A, Warming S, Larsen L, et al. Treatment of myofascial trigger-points with ultrasound combined with massage and exercise-a randomised controlled trial. Pain. 1998;77:73-9.

62. Gam A, Johannsen F. Ultrasound therapy in musculoskeletal disorders: a meta-analysis. Pain. 1995;63:85-91.

63. Aguilera FJ, Martín D, Masanet R, Botella A, Soler L, Morell F. Immediate effect of ultrasound and ischemic compression techniques for the treatment of trapezius latent myofascial trigger points in healthy subjects: a randomised controlled study. J Manipulative Physiol Ther. 2009;32:515-20.

64. Srbely J, Dickey J, Lowerison M, Edwards A, Nolet P, Wong L. Stimulation of myofascial trigger points with ultrasound induces segmental antinociceptive effects: a randomized controlled study. Pain. 2009;139:260-6.

65. Ay S, Doğan SK, Evcik D, Baser O. Comparison the efficacy of phonophoresis and ultrasound therapy in myofascial pain syndrome. Rheumatol Int. 2011;31:1203-8.

66. Sarrafzadeh J, Ahmadi A, Yassin M. The effects of pressure release, phonophoresis of hydrocortisone, 
and ultrasound on upper trapezius latent myofascial trigger point. Arch Phys Med Rehabil. 2012;93:72-7.

67. Majlesi J, Unalan H. High-power pain threshold ultrasound technique in the treatment of active myofascial trigger points: a randomized, double-blind, case-control study. Arch Phys Med Rehabil. 2004;85:833-6.

68. Crockett D, Foreman M, Alden L, Blasberg B. Comparison of treatment modes in the management of myofascial pain dysfunction syndrome. Biofeedback Self Regul. 1986;11:279-91.

69. Graff-Radford S, Reeves J, Baker R. Effects of transcutaneous electrical nerve stimulation on myofascial pain and trigger point sensitivity. Pain. 1989;37:1-5.

70. Hsueh C, Cheng P, Kuan T, Hong C. The immediate effectiveness of electrical nerve stimulation and electrical muscle stimulation on myofascial trigger points. Am J Phys Med Rehabil. 1997;76:471-6.

71. Lee J, Lin D, Hong C. The effectiveness of simultaneous thermotherapy with ultrasound and electrotherapy with combined AC and DC current on the immediate pain relief of myofascial trigger points. J Musculoskelet Pain. 1997;5:81-90.

72. Farina S, Casarotto $M$, Benelle $M$, et al. A randomized controlled study on the effect of two different treatments (FREMS and TENS) in myofascial pain syndrome. Eura Medicophys. 2004;40:293-301.

73. Gül K, Onal SA. Comparison of non-invasive and invasive techniques in the treatment of patients with myofascial pain syndrome. Agri. 2009;21:104-21.

74. Chu J, Yuen K, Wang B, Chan R, Schwartz I, Neuhauser D. Electrical twitch-obtaining intramuscular stimulation in lower back pain: a pilot study. Am J Phys Med Rehabil. 2004;83:104-11.

75. Chu J, Schwartz I. eToims twitch relief method in chronic refractory myofascial pain (CRMP). Electromyogr Clin Neurophysiol. 2008;48:311-20.

76. Pujol J, Pascual-Leone A, Dolz C, Delgado E, Dolz J, Aldoma J. The effect of repetitive magnetic stimulation on localized musculoskeletal pain. Neuroreport. 1998;9:1745-8.

77. Smania N, Corato E, Fiaschi A, Pietropoli P, Aglioti $S$, Tinazzi M. Therapeutic effects of peripheral repetitive magnetic stimulation on myofascial pain syndrome. Clin Neurophysiol. 2003;114:350-8.

78. Smania N, Corato E, Fiaschi A, Pietropoli P, Aglioti S, Tinazzi M. Repetitive magnetic stimulation a novel therapeutic approach for myofascial pain syndrome. J Neurol. 2005;252:307-14.

79. Ceylan Y, Hizmetli S, Siliğ Y. The effects of infrared laser and medical treatments on pain and serotonin degradation products in patients with myofascial pain syndrome. A controlled trial. Rheumatol Int. 2004;24:260-3.

80. Gur A, Sarac A, Cevik R, Altindag O, Sarac S. Efficacy of $904 \mathrm{~nm}$ gallium arsenide low level laser therapy in the management of chronic myofascial pain in the neck: a double-blind and randomize-controlled trial. Lasers Surg Med. 2004;35:229-35.

81. Ceccherelli F, Altafini L, Lo Castro G, Avila A, Ambrosio F, Giron G. Diode laser in cervical myofascial pain: a double-blind study versus placebo. Clin J Pain. 1989;5:301-4.

82. Hakgüder A, Birtane M, Gürcan S, Kokino S, Turan F. Efficacy of low level laser therapy in myofascial pain syndrome: an algometric and thermographic evaluation. Lasers Surg Med. 2003;33:339-43.

83. Ilbuldu E, Cakmak A, Disci R, Aydin R. Comparison of laser, dry needling, and placebo laser treatments in myofascial pain syndrome. Photomed Laser Surg. 2004;22:306-11.

84. Dundar U, Evcik D, Samli F, Pusak H, Kavunca V. The effect of gallium arsenide aluminum laser therapy in the management of cervical myofascial pain syndrome: a double blind, placebo-controlled study. Clin Rheumatol. 2007;26:930-4.

85. Waylonis G, Wilke S, O'Toole D, Waylonis DA, Waylonis DB. Chronic myofascial pain: management by low-output helium-neon laser therapy. Arch Phys Med Rehabil. 1988;69:1017-20.

86. Altan L, Bingöl U, Aykaç M, Yurtkuran M. Investigation of the effect of GaAs laser therapy on cervical myofascial pain syndrome. Rheumatol Int. $2005 ; 25: 23-7$.

87. Thorsen H, Gam A, Svensson B, et al. Low level laser therapy for myofascial pain in the neck and shoulder girdle. A double-blind, cross-over study. Scand J Rheumatol. 1992;21:139-41. 